\title{
PRACTICAL PROBLEMS IN THE ANALYSIS OF CP STARS
}

\author{
Charles R. Cowley \\ University of Micnigan
}

Sveneric Johansson

Lunds Universitet

\begin{abstract}
Saturation and curve-of -growth effects remain a dominant source of errors in abundance work. We suggest that the use of low noise material will enable future users to work more easily with weak lines and avoid these difficulties. The method of wavelength coincidence statistics is reviewed within the context of line identifications and abundance work. This technique is readily implemented, and should be a standard part of the analytical spectroscopy of stars. Analysis of the ultraviolet spectra of CP stars is severely handicapped by missing atomic data. Synthetic spectra are displayed showing the possible effects of hitherto unknown lines on spectra of IUE resolution.
\end{abstract}

\section{INTRODUCTION}

This paper will be concerned with selected, practical difficulties related to the analysis of stellar spectra.

The general methods of analytical stellar spectroscopy were detailed by Unsöld (1955) in the second edition of his famous textbook. Apart from the methods of non-LTE, whose successes have been limited to special cases, the improvements of the past 30 years have been primarily related to the speed of computation now possible. Especially in the analysis of $\mathrm{CP}$ stars, it can be said that current techniques hardly reflect the improvement in quality that one might have expected from an additional three decades of experience.

One of the reasons for this is that the stellar spectroscopists who have gained analytical experience have not remained in this field, although many are still active in astronomy. The lessons learned through experience have consequently not been retained, and abundance workers have repeated many of the mistakes of the past.

\section{SATURATION PROBLEMS AND PALLIATIVE MEASURES}

Of all of the errors that have affected the analysis of CP stars, the most important have probably been related to saturation, or curve-of-growth effects. These problems are not necessarily removed by spectral synthesis, although clearly, the use of information on line shapes as well as total strengths can be an advantage.

It is probably recognized by everyone that the use of weak lines avoids most saturation problems, but in spite of this fact, even very recent work has made use of strong lines. Why is this so? The most common reason for the use of stronger lines is that the spectral dispersion is insufficient to allow one to measure the weak lines accurately.

In typical abundance studies, the number of truly weak lines is of ten extremely small. Even in cases where hundreds of lines from a given spectrum may have been measured there may be less than half a dozen that are clearly uninfluenced by curve-of -growth effects.

The decision of a research worker to proceed with material of admittedly low quality (low dispersion, high noise) is of ten made for a variety of reasons, that are outside of the domain of scientific judgement. The question of importance is whether there is an obvious way 
to relieve this situation, and we suggest that there is. The Peculiar Newsletter (see Hensberge and van Rensbergen 1985, henceforth, PN) publishes information on the availability of spectroscopic material at various observatories and from various research workers. Moreover, the PN of ten indicates that a worker is interested in sharing data. We suggest far too much analytical spectroscopy has been done with material inferior to that which might have been obtained from another astronomer or institution, simply by requesting it. In present times, it should be the simplest matter to borrow digitized intensity data on a magnetic tape.

Thus an early step in the analysis of a star should be a search of the literature for possible spectroscopic DATA, followed by an earnest attempt to obtain it. ONLY in the case where it is clear that new observations would be superior to those already made, or if the existing data is unavailable, need one apply for observing time to obtain new material.

It is now common to choose a microturbulence which yields no correlation of abundance with equivalent width. This technique has shortcomings. It must be recognized that the procedure in no way improves the abundances, which still depend on the weakest lines (and in rare instances on the very strong ones). Moreover, the accuracy with which a line can be measured degenerates rapidly as the strength of a line approaches the threshold of detectability. Thus, as we remarked many years ago, systematic errors that depend on the (magnitude of the) equivalent widths are to be expected (Cowley 1970 Section 4-13.1). They should be carefully investigated, but in practice, this may not be done. We suggest that it is more common to adjust the microturbulence in such a way that the systematic errors are not apparent.

All abundance workers are urged to make a careful consideration of possible systematic errors in their equivalent width measurements before arbitrarily adjusting the microturbulence parameter. Careful consideration should also be given the maximum line depths allowed by the models, vis-a-vis those observed in the star.

The systematic errors to be expected for weak lines will obviously be less serious, for a given line strength, the greater the signal-to-noise level. Every attempt should therefore be made to achieve signal-to-noise levels of at least 50 . Of course, the signal-to-noise level that is necessary to measure a $20 \mathrm{~mA}$ line with an uncertainty of, say, $10 \%$, varies with the $v . \sin (i)$ of the star.

It is well to recommend the use of weak lines, but one cannot find critically evaluated gf-values for many of the weaker lines. Cowley and Adelman (1983) have stressed the importance of the "critical evaluations" by people at the US National Bureau of Standards, for astronomical applications. Usually, one can find gf-values from Kurucz and Peytremann (1975, henceforth, KP) for weak iron-group lines, but the accuracy of these values for nonLS-coupling allowed transitions, is known to be questionable. A critical problem of current interest is the upper limit for the iron abundance in some CP stars. Can it be as high as 9.0 $(\log [\mathrm{H}]=12)$, as suggested by the work of Muthsam and Cowley (1984) and others? Must the theoreticians take this seriously? It turns out to be quite difficult to evaluate this matter because the critically evaluated lines of Fe II, are not weak, at least not in those stars with the putative high iron abundances.

The problem is well illustrated in Table 2 of Muthsam and Cowley. The truly weak lines of Fe II are $\lambda \lambda 4625.55$ and 4666.77 . The abundances from these lines are at the extreme upper limit of those listed for the Fe II lines. If uncertainties in the oscillator strengths or blends could be ignored, we would be forced to conclude that [Fe/H] must be AT LEAST as large as 9.0, and is quite possibly larger! At present, there does not appear to be a straightforward way for the astronomer to deal with this particular problem. We await more accurate calculations and measurements.

For many other atomic spectra, the critical evaluations of the NBS cover weak lines. Cowley and Adelman (1983) have suggested some additional sources of gf-values. 


\section{THE PROBLEM OF IDENTIFICATIONS}

Authors of books on stellar atmospheres have apparently assumed the problem of line identification is so simple that no detailed discussion is needed. This is ironical. The problems can of ten be so difficult that no satisfactory solution can be found.

Line identification in late-type stars with solar abundances is straightforward in the traditional $(\lambda \lambda 3500-6500)$ region, for all but the weakest features. With CP stars, and even for "normal" stars in the satellite ultraviolet, one finds identification problems that can be vastly more difficult. The level of difficulty can vary enormously from one $\mathrm{CP}$ star to another, but in the satellite ultraviolet, line-by-iine identification work is difficult or impossible, even for "normal" stars.

No stellar identification work is begun without some prior knowledge of what is present in the spectrum. Only a college freshman begins with the stellar wavelengths and the finding list from the Multiplet Tables. The identification worker has at his disposal - as a minimum - a knowledge of the strongest lines: the hydrogen lines, $\mathrm{H}$ and $\mathrm{K}$ of $\mathrm{Ca} \mathrm{II}$, etc. Usually, he has a list of identifications of wavelengths in some similar star to the one studied. Merrill's (1956) old book is still a usef ul compendium for work of this kind. The most usef ul sources for CP stars are unpublished studies by Bidelman $(1966,1968)$ who employed high dispersion spectra of stars with intrinsically sharp spectral lines. Of the published works, some of the more useful are those of Adelman (1974), Hiltner (1945), and the Jaschek's and González (1965). The researcher must keep in mind that considerable advances in atomic spectroscopy have been made since many of the older identification studies were done.

The identification of lines to use for abundances has of ten been made without measurements of wavelengths and we suggest that this can be a serious mistake. A measured wavelength that deviates by significantly more than the expected error is an immediate indication of a blend, and in rarer cases of isotope shif ts ( $\mathrm{Hg} \mathrm{II}, \mathrm{Pt} \mathrm{II})$. Fortran programs are available from a number of workers for the automatic measurement of digitized spectra, and for many $\mathrm{CP}$ stars digitized measurements are available on request (from $\mathrm{CRC}$ ) for a large number of late B and A stars. The abundance worker who fails to take advantage of this material is remiss.

Identifications for a given element must start with a search for the strongest expected lines. The lines may be judged to be "present" or below some threshold for detection. In the latter case it is possible to set an upper limit to the abundance. This upper limit is of ten uninteresting, because a rather excessive overabundance of some exotic species could still be possible. Nevertheless, it is better to determine such an upper limit than to say the lines are "absent."

The traditional method of line identifications relies heavily on the relative (laboratory) intensities within multiplets. It is usually assumed that the relative intensities within a multiplet are unaffected by non-thermal excitations. This is a good working assumption, although one should always be aware of the possibility of selective excitations (Johansson and Jordan 1984, Cowley 1970 Table 2-6.1).

The method of multiplets works very well in many instances, but there are certainly cases where other techniques are preferable. First, we note that multiplets are traditionally defined on the assumption of LS coupling, which can be a very bad approximation. When this is the case, one must beware of theoretical LS-coupling intensities. Second, in the kinds of spectra that occur with the rare earths, there are a large number of lines with similar intensities that arise from levels not very different in excitation. Under these circumstances, the intensities of Meggers, Corliss, and Scribner (1975) are probably as good as the relative intensities in an LScoupling approximation to a multiplet. These "natural" relative intensities should be useful even in the case of non-thermal excitations in the source. While the theoretical LS-coupling intensities may be perturbed by the mixing of (LS) states, laboratory intensities are not subject to this particular problem.

Neither the method of multiplets, nor any other method is of much use when the 
strongest expected lines of a given species are only weakly present. The argument that there is no other candidate for the identification of a given feature other than the strongest line of, say $X$, has little weight unless it is possible to securely identify virtually all of the lines in the spectrum apart from the one(s) in question.

In a heavily blended spectrum, the relative intensities can be so badly distorted, that even rather good laboratory data on line strengths can be of little use. If a line to be identified is in a multiplet, one must of course check to see if stronger lines in the multiplet are present with the proper intensities. Such a check should be made as a matter of course, but much of the power of the method is lost if all of the lines in question are rather weak, and the relative intensities vary only by a factor of two or three. When the candidate line is itself the strongest line in a multiplet, but it is very weak, it is rather meaningless to search for its congeners in a noisy spectrum.

C. Cowley and his collaborators have employed the technique of wavelength coincidence statistics (WCS). It is a global method, which gives no direct information on individual features, but provides an impersonal basis for rejection of the null hypothesis, that lines of given ion are not present in the stellar spectrum. Rejection of the null hypothesis at a high confidence level means (within a calculable risk) that an ion is present. Failure to reject the null hypothesis does not prove the absence of a species, which may be weakly present.

WCS has been applied with good success to high dispersion spectra $(\mathrm{ca} .2 \mathrm{~A} / \mathrm{mm})$ in the region $\lambda \lambda 3000-6600$ (see Cowley and Hensberge 1981). Limited applications have been made in the deeper UV (Hensberge et al. 1986, Bord and Davidson 1982, Davidson and Bord 1982).

Recent applications of WCS have incorporated information on intensities (Chjonacki, et al. 1984, Henseberge, et al. 1986). While the value of this information is more limited than one might think, important conclusions can be drawn from intercomparisons of the intensity levels at which sets of lines with different average intensities reach a given level of significance in stars with similar line widths $(v \sin (i)$ 's) and line densities. Likewise, one may intercompare the WCS parameters for the spectra of two elements in a given star; in such a comparison, one has the advantage that the line widths and densities are essentially constant. In the lanthanides, we usually employ three lists, a strong, an intermediate, and a weak set. In the spectrum of HR 7575 and $\beta \mathrm{CrB}$, the WCS parameters for Nd II and Sm II indicate only marginal significances for all three lists, while for $\gamma$ Equ, the WCS parameters are highly significant, for all three sets. Moreover, for La II, Ce II, and Gd II, the WCS parameters are highly significant in both stars. This result has enabled us to conclude that there is a "hole" in the lanthanide abundance distribution for the former stars, since we can think of no effect related to their spectra, such as line density, or broadening, that could account for such an apparently selective effect.

There are numerous ways of making line identifications. Each method has special advantages and disadvantages, many of which have been outlined above. The competent research worker selects those methods that are the most powerful for his special problems, and applies them with insight into their strengths and weaknesses. We recommend that WCS be employed at an early stage in any attempt at a line-by-line identification study, and that the worker look first for those species for which the WCS parameters indicate the presence beyond any reasonable doubt. Such elements will be typically from the iron-group, to be sure, but the clear presence of more exotic species may also be indicated.

For most of the iron-group elements, it would not be difficult to prepare a list of lines with intensity estimates made from gf -values, and to simply proceed with identifications down to a given threshold. Especially for the rare earths, the rough gf-values obtained from Monograph 145 (Cowley and Corliss 1983) should prove to be of value.

\section{MISSING ATOMIC DATA, AND THE SATELLITE UV}

It is generally well understood by astronomers that there is a pressing need for physical data on gf-values, collision cross sections, and line-broadening parameters. It is less of ten 
recognized that important work still needs to be done in the more basic domains of atomic structure-wavelengths and energy levels. This need has been demonstrated by various studies over the last decade, but the point still needs to be emphasized. The long path lengths and high temperatures of stellar atmospheres create powerf ul light sources that are difficult to simulate in the laboratory.

For ions, this problem is critical, because the densities are not high enough to allow absorption measurements. Absorption measurements have been made, of course, for neutral atoms. Thus in stellar photospheres, we "measure" the population of the lower level of a transition, while in the laboratory, one may see the corresponding line if one can manage to get sufficient population of the upper level. In practice, this means that for stars with high excitation temperatures, we will expect some unidentified lines, because the conditions for observing them in the laboratory are much less favorable than in the star.

We have referred to faint, high-excitation lines as "second generation" lines, because they do not appear in the classical lines lists such as the Multiplet or MIT Wavelength Tables. In fact, the Multiplet Tables do contain lines predicted on the basis of known (circa 1945) energy levels, mostly in Fe I. The second generation lines with which we are primarily concerned with here arise from levels that have been found in the laboratory within, say, the past decade.

Cowley and Arnold (1978) used WCS to demonstrate the presence of newly predicted lines in the region $\lambda \lambda 3750-4650$ of $C P$ stars, and pointed out that chance coincidences with such lines have caused considerable confusion. The use of the tables of KP and their congeners (e.g. Kurucz 1981, henceforth, K-81) can eliminate many of the problems caused by such lines, but the tables that have been published as of this writing, were constructed before much of the recent work on the high level structure of iron-group spectra (see Johansson 1981). The KP Tables include only the Corliss-Bozman (1962) wavelengths for spectra of elements heavier than nickel, and therefore lack many of the important additions, especially in the rare earth spectra incorporated into in NBS Monograph 145, and in Atomic Energy Levels-The Rare Earths (Martin, Zalubas, and Hagan 1978).

The problem of second generation lines takes on an entirely new dimension when we consider the satellite ultraviolet, extensively studied now at high resolution for a decade with the help of the International Ultraviolet Explorer (IUE). In. the near future, the more powerful instrumentation of the Space Telescope will be available, and the time is ripe for an examination of the question of how seriously handicapped we are by our present, incomplete knowledge of the high level structure, especially of iron-group elements.

We discuss here a preliminary study of a $5 \mathrm{~A}$ region centered at $\lambda 1850$, which was made to evaluate the importance of second-generation spectra on a study such as that of Leckrone (1984) of the $\mathrm{Hg}$ II line at $\lambda 1942$. All calculations are based on a stellar model with $\mathrm{T}(\mathrm{eff})=$ $11000 \mathrm{~K}$, and $\log (\mathrm{g})=3.5$. The assumed abundances throughout are solar except for chromium, manganese iron, which are all enhanced by 1.0 dex. The enhancement for iron is greater than in a typical $\mathrm{Hg}-\mathrm{Mn}$ star, but the difference should not be important in the present context, which is primarily illustrative. We-also calculate strengths for a resonance line of a hypothetical element whose properties (ionization energies, partition functions, etc.) are those of mercury ( $\mathrm{Hg}$ II $\lambda 1942$ ) except that we assume a rest wavelength of $\lambda 1850.00$. This wavelength was chosen before the lines within it were investigated. It turned out by chance that some important absorption by second-generation features fell very close to the rest wavelength assumed for the assumed line (hypotheticum: Hy II $\lambda 1850.00$ ).

Figure 1 shows the region with only lines from the KP tables. In Figure 2, we give results with only second generation lines of $\mathrm{Cr}, \mathrm{Mn}$, and $\mathrm{Fe}$. Details concerning these lines will be published elsewhere, but for the present, we note that these particular wavelengths all represent transitions between recently classified energy levels (Johansson 1986). Oscillator strengths were estimated, roughly, from the laboratory intensities. The theoretical spectra include neither instrumental effects nor rotational broadening, but a microturbulence of $2 \mathrm{~km} /$ sec was assumed. 


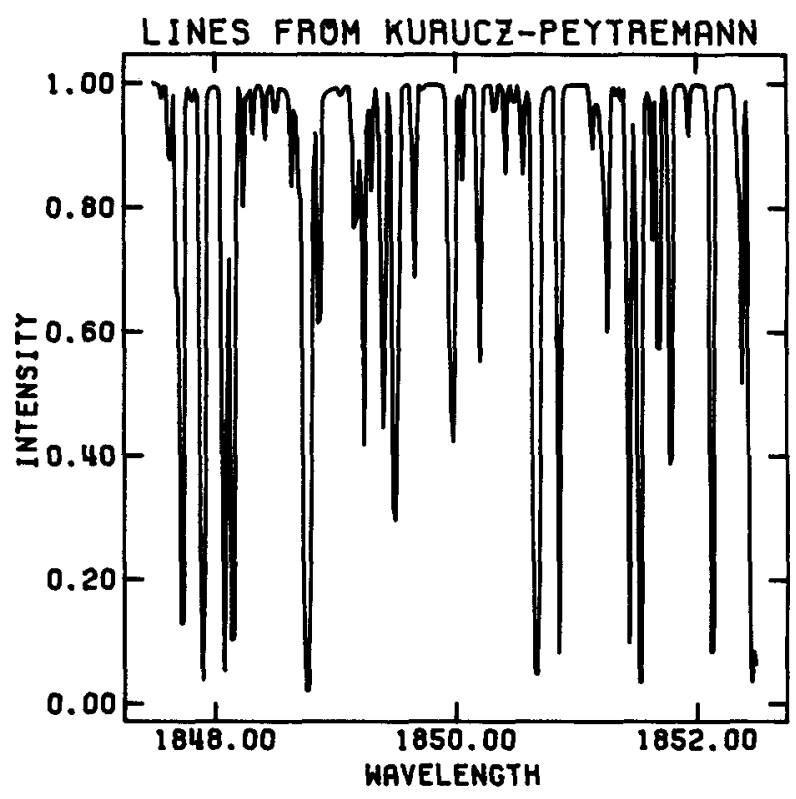

Figure 1. Theoretical spectrum KP lines: $W_{\lambda}=889 \mathrm{~mA}$.

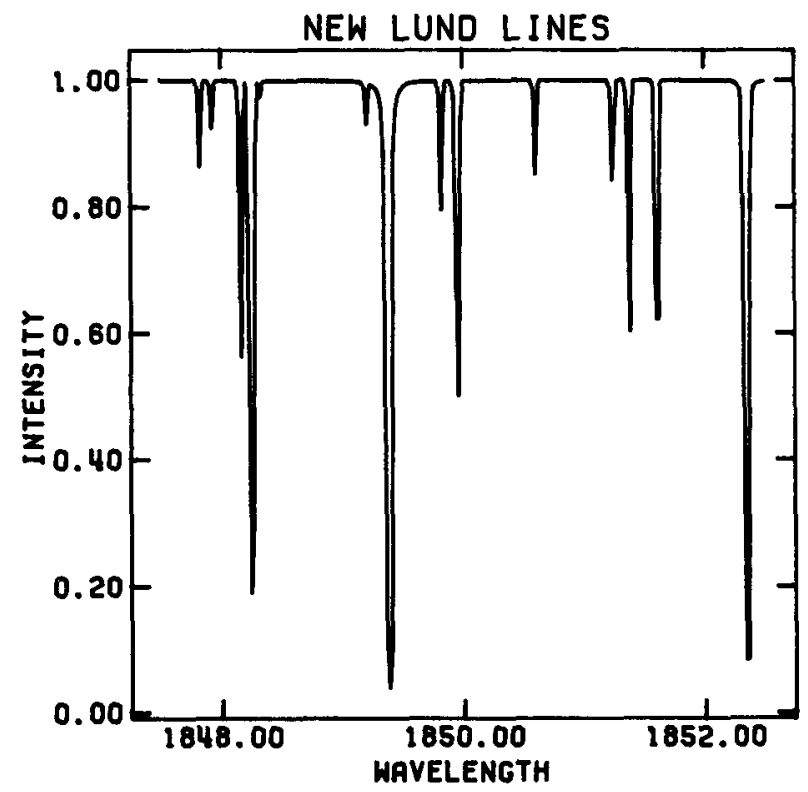

Figure 2. Theoretical spectrum including only new lines from Lund: $\mathrm{w}_{\lambda}=$ $228 \mathrm{~mA}$.

The second-generation lines provide a non-negligible absorption, although because of 
saturation effects, the combined absorption ( $988 \mathrm{~mA}$, not illustrated) is increased only by about $11 \%$.

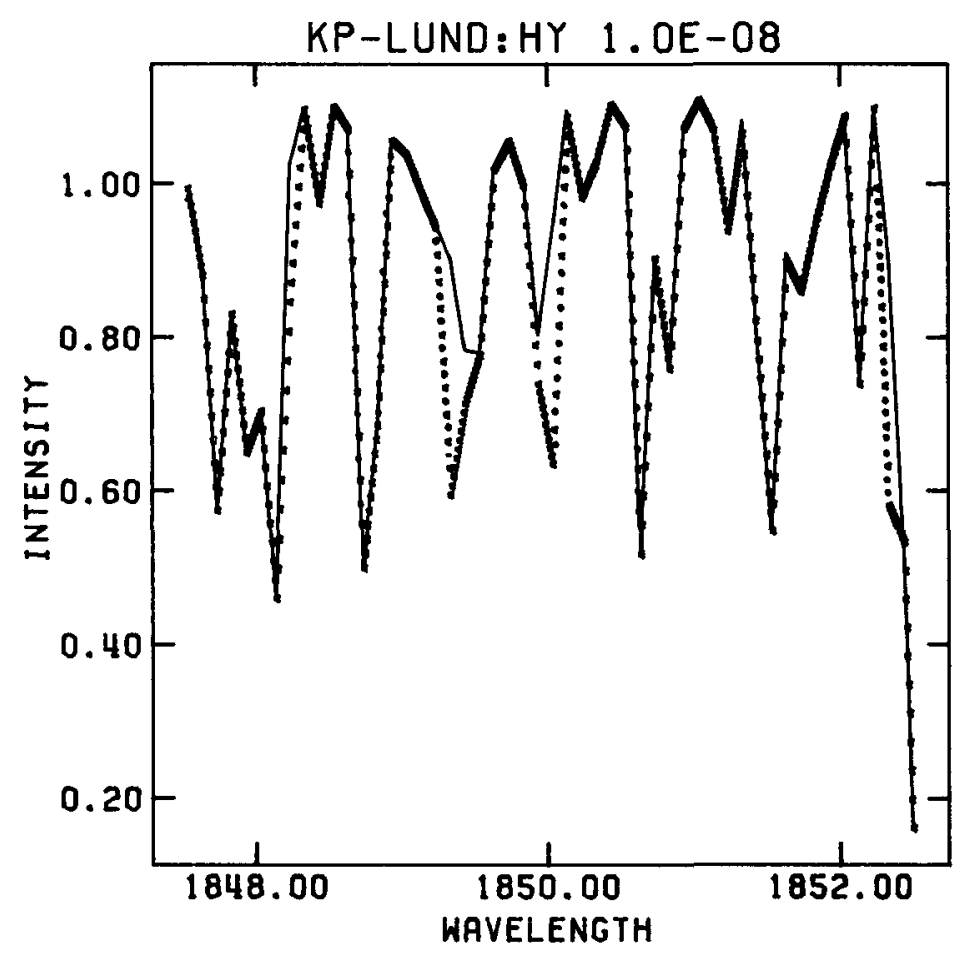

Figure 3. Simulated IUE spectra in the Hy II region. The Hy abundance fraction assumed is $10-8$, corresponding to an excess of 100 (if $\mathrm{Hy}$ were $\mathrm{Hg}$ ). The solid curve includes only lines from KP and Hy II. The points represent the additional contribution from second-generation lines.

Kurucz (K-81) updated the KP calculations for the Fe II spectrum only. His work rested primarily on analyses at Lund (see Johansson 1978), supplemented by purely theoretical calculations. The additional (non-KP) Fe II lines in $\lambda \lambda 1847.5-1852.5$ give a total absorption of $0.13 \mathrm{~A}$. They are comparable in importance to the absorption from the second-generation lines of our Figure 2, although somewhat smaller. It is important to note that the majority of the absorption from these $\mathrm{K}-81$ lines comes from a set of lines near $\lambda 1848.5$ for which the upper levels were not correctly classified in $\mathrm{K}-81$. The correct amount of absorption from these particular levels is in reality much lower. This represents an unusual example where an astronomer might overestimate the contribution from Fe II.

We have made a number of sample calculations with various $\mathrm{Hy}$ abundances, including and excluding the second-generation lines. The calculated spectra were subjected to a $0.1 \mathrm{~A}$ binning, with $15 \%$ noise added to simulate IUE spectra. We recall that Leckrone finds excesses of $\mathrm{Hg}$ of the order of $10^{4}$. If we adopt a corresponding abundance for $\mathrm{Hy}$, the resulting $\mathrm{Hy} \mathrm{II}$ line is sufficiently strong that it is relatively unperturbed by the presence of the additional second-generation lines. However, for an Hy abundance roughly $10^{2}$ that of (the solar value of $\mathrm{Hg}$ ), the relative importance of the new lines is perceptible, as is shown in Figure 3.

It appears that Leckrone's conclusions regarding mercury excesses should be 
substantially correct, at least as far as the second-generation lines are concerned. When the $\mathrm{Hg}$ excesses are rather smaller than in the stars he investigated, more attention will have to be given to missing atomic data. We must keep in mind, of course, that in a different wavelength region, the second generation absorption could be substantially more, (or less).

\section{REFERENCES}

Adelman, S. J. 1974, Ap. J. Suppl., 28, 51.

Bidelman, W. P. 1966, in Abundance Determinations in Stellar Spectra., IAU Symposium 26, p. 229.

Bidelman, W. P. 1968, in Nucleosynthesis, ed. W. D. Arnett, C. J. Hansen, J. W. Truran, and A. G. W. Cameron (New York: Gordon and Breach), p. 63.

Bord, D. J., and Davidson, J. P. 1982, Ap. J., 258, 674.

Chjonacki, G. T., Cowley, C. R., and Bord, D. J. 1984, Ap. J., 286, 736-746.

Corliss, C. H. and Bozman, W. R. 1962, N. B. S. Monograph,

Cowley, C. R. 1970, The Theory of Stellar Spectra, (New York: Gordon and Breach).

Cowley, C. R., and Adelman, S. J. 1983, Quart. Journ. Roy. Astron. Soc., 24, 393.

Cowley, C. R., and Arnold, C. N. 1978, Ap. J., 226, 420.

Cowley, C. R., and Corliss, C. H. 1983, Mon. Not. Roy. Astron. Soc., 203, 651.

Cowley, C. R., and Hensberge, H. 1981, Ap. J., 244, 252.

Davidson, J. P., and Bord, D. J. 1982, Astron. Ap., 111, 362.

Henseberge, H., and van Rensbergen, W. 1985, A Peculiar Newsletter, Fourteenth issue, July 1.

Hensberge, H., Van Santvoort, J., van der Hucht, K. A., and Morgan, T. H., 1986, Astron. Ap., in press.

Hiltner, W. A. 1945, Ap. J., 102, 438.

Jaschek, M., Jaschek, C., and González, Z. 1965, Zs. f. Ap., 62, 21.

Johansson, S. 1978, Physica Scr., 18, 217.

Johansson, S. 1981, in 23rd Liège International Astrophysical Colloquium, Upper Main Sequence Chemically Peculiar Stars, (Liège: Institut d'Astrophysique), p. 229.

Johansson, S. 1986, in preparation.

Johansson, S., and Jordan, C. 1984, Mon. Not. Roy. Astron. Soc., 210, $239-256$.

Kurucz, R. L. 1981, Smith. Ap. Obs. Spec. Rept., 390,(K-81).

Kurucz, R. L., and Peytremann, E. 1975, Smith. Ap. Obs. Spec. Rept., 362, (KP).

Leckrone, D. S. 1984, Ap. J., 286, 725.

Martin, W. C., Zalubas, R., and Hagen, L. 1978, Atomic Energy Levels-The Rare Earths, NBS-NSRDS, 60, (AE IV).

Meggers, W. F., Corliss, C. H., and Scribner, B. F. 1975, NBS Monograph, 145.

Merrill, P. W. 1956, Lines of the Chemical Elements in Astronomical Spectra, Carnegie Institution of

Muthsam, H., and Cowley, C. R. 1984, Astron. and Ap., 130, 348.

Unsöld, A. 1955, Physik der Sternatmosphären, 2nd Ed., (Berlin: Springer-Verlag).

Discussion appears after the following paper. 